\title{
Polysaccharide food matrices for controlling the release, retention and perception of flavours
}

Article

Accepted Version

Creative Commons: Attribution-Noncommercial-No Derivative Works 4.0

Cook, S. L., Methven, L., Parker, J. K. ORCID:

https://orcid.org/0000-0003-4121-5481 and Khutoryanskiy, V.

V. (2018) Polysaccharide food matrices for controlling the release, retention and perception of flavours. Food Hydrocolloids, 79. pp. 253-261. ISSN 0268-005X doi: https://doi.org/10.1016/j.foodhyd.2017.12.023 Available at https://centaur.reading.ac.uk/74664/

It is advisable to refer to the publisher's version if you intend to cite from the work. See Guidance on citing.

Published version at: https://www.sciencedirect.com/science/article/pii/S0268005X17313346

To link to this article DOI: http://dx.doi.org/10.1016/j.foodhyd.2017.12.023

Publisher: Elsevier

All outputs in CentAUR are protected by Intellectual Property Rights law, including copyright law. Copyright and IPR is retained by the creators or other copyright holders. Terms and conditions for use of this material are defined in the End User Agreement. 


\section{CentAUR}

Central Archive at the University of Reading

Reading's research outputs online 
1 Polysaccharide food matrices for controlling the release, retention and

2 perception of flavours

3 Sarah L. Cook a, Lisa Methven ${ }^{b}$, Jane K. Parker ${ }^{b}$, Vitaliy V. Khutoryanskiy* a

4 a Department of Pharmacy, University of Reading, Whiteknights, PO box 224, Reading,

5 Berks, RG6 6AD, United Kingdom

6 b Department of Food and Nutrition Sciences, University of Reading, Whiteknights, Reading,

7 Berks, RG6 6AD, United Kingdom

8 * Correspondence to: Professor V Khutoryanskiy Department of Pharmacy, University of 9 Reading, Whiteknights, Reading, Berks, RG6 6AD, United Kingdom. $10 \quad$ v.khutoryanskiy@reading.ac.uk

Abstract

13 Polysaccharides have many roles across both the food and pharmaceutics

14 industries. They are commonly used to enhance viscosity, stabilise emulsions 15 and to add bulk to food products. In the pharmaceutics industry, they are also 16 utilised for their mucoadhesive nature. Mucoadhesive polysaccharides can 17 facilitate retention of active ingredients at mucosal sites for a prolonged time and formulations can be designed to control their release and bioavailability.

19 This study investigates how polysaccharides, with differing physicochemical 20 properties (e.g. functional groups and molecular weight), affect the release 21 and perception of flavour compounds from films. Polysaccharide films were 22 prepared using either high or low viscosity carboxymethyl cellulose, pullulan or hydroxypropyl methylcellulose. Glucose, vanillin or a combination of both

24 was also added to the films to assess the effect of flavour release and 25 perception over time. The films were assessed for glucose release in vitro, 26 swelling and disintegration times, and mucoadhesive ability. Results show 27 that flavour release and perception depend on the polysaccharide matrix properties; this includes how quickly the films dissolves, the rate of release of 
29 tastant compounds, and the mucoadhesive strength of the polysaccharide. A 30 higher viscosity and slower disintegration time resulted in slower release of 31 glucose in vitro and flavour perception in vivo.

Key words: polysaccharides, flavour, controlled, release, mucoadhesion

1. Introduction

Flavour perception requires the release of taste and aroma compounds from the food matrix and the subsequent transport of those compounds to the respective receptors. This process is dependent on many factors including the properties of the compound, the components of the food matrix constituents, 40 food structure, how it is manipulated in the mouth and the physiological 41 conditions of the mouth, nose and throat during consumption of the food. 42 Furthermore, the onset and duration of flavour delivery is dependent on 43 factors such as partitioning, mass transport and diffusion. These factors play 44 varying roles and combined, result in a characteristic flavour profile for a food. 45

46 Typically, the polysaccharides, proteins and fats present in liquid food 47 systems determine the structure. The influence of these large molecules on 48 smaller molecules, such as aroma and tastant compounds, has been 49 investigated with various studies concluding that viscosity changes 50 (Hollowood, Linforth, \& Taylor, 2002; Izutsu, Taneya, Kikuchi, \& Sone, 1981; 51 Kokini, Bistany, Poole, \& Stier, 1982; Secouard, Malhiac, Grisel, \& Decroix, 52 2003; Stevenson \& Mahmut, 2011) and physical entrapment of compounds 53 (Keršiene, Adams, Dubra, Kimpe, \& Leskauskaite, 2008; Kora, Souchon, 
54 Latrille, Martin, \& Marin, 2004; Kuo \& Lee, 2014) together explain perceptual

55 differences (S. L. Cook, Bull, Methven, Parker, \& Khutoryanskiy, 2017). These

56 studies tend to focus on the matrix structure and the release characteristics

57 when contemplating changes in perception.

58

59 Chemical interactions between the flavour compounds and the food matrix is 60 also important (Heilig, Heimpel, Sonne, Schieberle, \& Hinrichs, 2016;

61 Rodríguez-Bencomo et al., 2011; Scherf, Pflaum, Koehler, \& Hofmann, 2015).

62 Factors such as charge of the flavour compound and other food constituents

63 will influence interactions between the two. For example, sodium is positively 64 charged and will therefore interact with negatively charged polysaccharides, such as carboxymethyl cellulose, affecting the ions availability to elicit a salt taste (Scherf et al., 2015). Retention of flavour compounds in the matrix will obviously decrease their perception, as they will not reach the respective receptors to be perceived and risk being swallowed in the food bolus before triggering perception. However, if the matrix also adheres to the oral mucosa then fewer tastant molecules may be swallowed allowing for release of the flavour over time.

Many studies have investigated the impact on aroma release when reducing fat in foods (Arancibia, Jublot, Costell, \& Bayarri, 2011; Bayarri, Taylor, \& Hort, 2006). They have found, in general that aroma retention in the matrix of a high fat food will increase as the $P$ (partition coefficient of a molecule between a lipophillic and an aqueous phase, usually octanol and water, respectively) of the aroma compound increases. This means it will favour 
79

80

81

82

83

84

85

86

87

88

89

90

91

92

93

94

95

96

97

98

99

100

101

102

103

being in the fatty matrix over partitioning into the aqueous saliva. Hydrophilic compounds ( $\log \mathrm{P}$ equal to or less than zero) on the other hand tend to be less dependent on changing fat levels (Arancibia, Castro, Jublot, Costell, \& Bayarri, 2015; Arancibia et al., 2011). In low fat systems, the release of hydrophobic aromas will be faster leading to an unbalanced flavour profile.

(1)

More recently, interactions between food components and the oral and nasal mucosa have been investigated. Specifically, interactions between flavour molecules and the oral mucosa may explain persistence of aromas in certain foods (Esteban-Fernández, Rocha-Alcubilla, Muñoz-González, MorenoArribas, \& Pozo-Bayón, 2016; Sánchez-López, Ziere, Martins, Zimmermann, \& Yeretzian, 2016). Furthermore, interactions between food matrices and the oral mucosa have been of interest with regard to negative sensory characteristics of dairy products (Bull et al., 2015; Hilal Y et al., 2015; Withers, Cook, Methven, Godney, \& Khutoryanskiy, 2013) and the impact of fat reduction on perception of foods (De Hoog, Prinz, Huntjens, Dresselhuis, \& Van Aken, 2006; Dresselhuis, van Aken, de Hoog, \& Martien, 2008). (1)

.

Many polysaccharides are mucoadhesive, meaning they adhere to mucosal surfaces in the body via intermolecular forces (hydrogen bonding, electrostatic attraction, hydrophobic interactions and covalent bonds) and physical penetration and entanglement of polymer chains (Andrews, Laverty, \& Jones, 2009; Huang, Leobandung, Foss, \& Peppas, 2000; Jabbari, Wisniewski, \& Peppas, 1993). Though this phenomenon has been of interest and well utilised in the pharmaceutics field for decades, the importance in the food 
104 industry is beginning to gain interest (Bull et al., 2015; S. L. Cook, Bull, et al., 105 2017; S. L. Cook, Woods, Methven, Parker, \& Khutoryanskiy, 2018; Gibbins \&

106 Carpenter, 2013; Hilal Y et al., 2015; Malone, Appelqvist, \& Norton, 2003;

107 Withers et al., 2013).

109 Mucoadhesive polymers can retain and control the release of active 110 pharmaceutical ingredients (APIs) at mucosal surfaces including those in the 111 oral cavity (Andrews et al., 2009). The mechanisms of mucoadhesion have 112 been described in the literature numerous times (Peppas \& Huang, 2004; 113 Shaikh, Singh, Garland, Woolfson, \& Donnelly, 2011; Smart, 2005, 2014). The 114 physicochemical interactions depend on the polymeric substance (e.g ionic 115 groups, chain length), the state of hydration of the polymer, the mucosal 116 secretions (e.g. $\mathrm{pH}$, thickness, mucin concentration) and the epithelial 117 structure and morphology (e.g. roughness and presence of micro cracks). The 118 fact that mucoadhesive polymers can retain small molecules at mucosal 119 surfaces and control their release will be important for the food industry to 120 consider as these frequently used polysaccharides may also retain tastant 121 and aroma molecules in a similar way (S. L. Cook, Woods, et al., 2018).

123 Many polysaccharides used in the food industry that are also mucoadhesive 124 include, but are not limited to; carboxymethyl cellulose (Yehia, El-Gazayerly, 125 \& Basalious, 2008, 2009), sodium alginate (Juliano, Gavini, Cossu, Bonferoni, 126 \& Giunchedi, 2004; Richardson, Dettmar, Hampson, \& Melia, 2004) and 127 pectin (Kaur \& Kaur, 2012; Thirawong, Nunthanid, Puttipipatkhachorn, \& 128 Sriamornsak, 2007). Buccal films are a formulation type made by dissolving a 
129 polymer in a solvent, adding the API and evaporating the solvent to leave a

130 thin film of polymer matrix containing the API (Gherman, Zavastin, Ochiuz,

131 Biliuta, \& Coseri, 2016; Kaur \& Kaur, 2012; Satishbabu \& Srinivasan, 2008;

132 Semalty, Semalty, Kumar, \& Juyal, 2008). Buccal films can be designed to

133 release API over differing periods of time.

134

135 The only study investigating the effect of mucoadhesive polysaccharides on

136 flavour retention and perception was within an aqueous system. Also from our

137 group, our findings suggest that sodium ions are retained in the mouth for

138 longer when mucoadhesive polysaccharide is used as a thickener compared

139 to non-mucoadhesive matrices (S. L. Cook, Woods, et al., 2018). This current 140 study is concerned with the effect of mucoadhesive polysaccharides on

141 flavour perception from a solid food system (films). Various food grade

142 polysaccharides that differ in their chemical and physical properties were used

143 to assess the effect on release, retention and perception of flavours from 144 polysaccharide films.

146 Polysaccharides were cast into films containing glucose and/or vanillin.

147 These were based on films usually made for pharmaceutical applications. The 148 mucoadhesive properties, swelling ratio, dissolution rate, film thickness, water 149 activity and temporal sensory perception were assessed. Whilst this study 150 takes those factors into consideration, a further interaction between the food 151 matrix and the oral anatomy, mucoadhesion, is investigated. The aim for this 152 study was to assess the differences in flavour release from different 153 polysaccharide matrices in a solid state. It was hypothesised that films made 
154 with more viscous, slower dissolving polysaccharides will reduce the intensity 155 but prolong the perception of flavours over time. Furthermore, the 156 mucoadhesive properties of the matrices were assessed and related to 157 flavour delivery. This study, therefore, provides a foundation of understanding 158 of the mechanisms by which mucoadhesive ingredients can alter the 159 perception of flavour over time, which may help in the development of 160 reformulated products.

\section{2. Methods}

163 2.1. Materials

164 Four different polysaccharides were chosen for this study due to their differing 165 chemical properties (Table 1). Pullulan (PUL) (Hayashibara nagase europa 166 group, Düsseldorf, Germany) was chosen as a non-ionic, low viscosity and 167 fast dissolving film former. Hydroxypropyl methyl cellulose (HPMC) (product 168 code METHOCEL K4M, Dow The Chemical Company, Staines, UK) was 169 chosen as a high viscosity, non-ionic film former. Two carboxymethyl cellulose 170 products were used, one low molecular weight (LCMC) (product code 171 AKUCELL AF 0305, AkzoNoble, Amsterdam, The Netherlands) and one high 172 molecular weight (HCMC) (product code WALOCEL 4500, Dow The Chemical 173 Company, Staines, UK). Carboxymethyl cellulose was chosen as it is well 174 known for its mucoadhesive properties due to its ionic nature and high 175 viscosity. 
Table 1. Polysaccharide characteristics

\begin{tabular}{lllll}
\hline Sample & Molecular & Sodium & Degree of & Viscosity of 2\% \\
& weight (Da) & content $(\%$ & substitution & $(\mathrm{w} / \mathrm{v})$ solution at \\
& & $\mathrm{w} / \mathrm{v})$ & $25^{\circ} \mathrm{C}(\mathrm{mPa} . \mathrm{s})$ \\
\hline PUL & 250,000 & $<0$ & $\mathrm{~N} / \mathrm{A}$ & 11 \\
LCMC & 140,000 & $15.4^{*}$ & 0.8 & 450 \\
HPMC & 300,000 & $<0$ & $1.8 \quad$ methoxyl & 4500 \\
& & & 0.13 & \\
& & & hydroxypropyl & \\
HCMC & 950,000 & 8.7 & 0.8 & 5200
\end{tabular}

All data provided by the respective manufacturer except those indicated by *.

181 * Sodium content determined by flame photometry

\subsection{Samples}

184 Films were prepared by dissolving polysaccharides in deionised water (2\% w/v) with glucose, vanillin (Sigma- Aldrich, St. Louis, Missouri, United States)

186 or glucose and vanillin (Table 2). The solution $(30 \mathrm{~g})$ was weighed into circular 187 petri dishes $(90 \mathrm{~mm})$ and placed in an oven at $65^{\circ} \mathrm{C}$ for 20 hours. Once the 188 films were dry they were removed from the petri dish and cut into squares 189 (approx. $1 \mathrm{~cm}^{2}$ ). Glucose containing films weighed $100 \mathrm{mg}$ and the aroma only 190 films $30 \mathrm{mg}$. This was to ensure that each sample contained the same amount 191 of polysaccharide. The water activity $\left(a_{w}\right)$ of the films was measured after the 192 drying process using a HygroLab C1 Bench-Top Water Activity Monitor. 
195 Table 2. Final concentrations of ingredients in each type of film

\begin{tabular}{lccc}
\hline Film type & Polysaccharide (\%) & $\begin{array}{c}\text { Glucose } \\
(\% w / v)\end{array}$ & Vanillin (\%) \\
& 30 & 70 & - \\
\hline Sweet & 99.1 & - & 0.9 \\
Vanilla & 29.5 & 69.4 & 0.9 \\
Sweet and Vanilla & & & \\
\hline
\end{tabular}

196

2.3. Artificial saliva

198 Artificial saliva (AS) was used for all in vitro experiments to emulate conditions 199 in the mouth. This was adapted from Madsen et al. (2013) and consisted of $200 \quad 0.21 \mathrm{~g} / \mathrm{L} \mathrm{NaHCO}_{3}, 0.43 \mathrm{~g} / \mathrm{L} \mathrm{NaCl}, 0.75 \mathrm{~g} / \mathrm{L} \mathrm{KCl}, 0.22 \mathrm{~g} / \mathrm{L} \mathrm{CaCl} \mathrm{C}_{2} \cdot 2 \mathrm{H}_{2} \mathrm{O}, 0.91 \mathrm{~g} / \mathrm{L}$ $201 \mathrm{NaH}_{2} \mathrm{PO}_{4} \cdot 2 \mathrm{H}_{2} \mathrm{O}$ dispersed in deionized water. For the mucoadhesion 202 experiment $2.5 \mathrm{~g} / \mathrm{L}$ pig gastric mucin (PGM) type II (Sigma- Aldrich, St. Louis, 203 Missouri, United States) was also added. The $\mathrm{pH}$ of the AS was adjusted to 2046.8 and kept at $37^{\circ} \mathrm{C}$ during experiments and at $4{ }^{\circ} \mathrm{C}$ when not in use.

205

\subsection{Swelling and disintegration}

207 Swelling studies were carried out in an incubator set to $37^{\circ} \mathrm{C}$. Each film was 208 placed on to netting and fully submerged in a petri dish with $40 \mathrm{~mL}$ of $\mathrm{AS}$. At 209 set time periods the sample was removed from the AS, excess water was 210 carefully absorbed with tissue paper and the film on the netting was weighed.

211 This process was repeated until the weight had returned to that of the netting 212 alone. Each type of film was tested 6 times with duplicate batch repeats. Film 213 thickness was measured before these experiments with a micrometer. The 
214 maximum swelling ratio was determined by dividing the weight of the film at

215 set time points with the original weight of the film.

\subsection{Dissolution}

218 Each film containing glucose was placed onto netting and carefully 219 submerged into an individual beaker with $200 \mathrm{~mL}$ AS. The solution was stirred 220 by a magnetic stirrer bar at a constant rate throughout the experiment. At set 221 time points $1 \mathrm{~mL}$ aliquots of the $\mathrm{AS}$ medium were removed and put into 222 labelled Eppendorfs for analysis. The glucose in the samples was quantified 223 spectrophotometrically using an Amplex Red, glucose oxidase kit following 224 the advised protocol (Fisher Scientific, Loughborough, UK). Each sample was 225 tested 6 times with duplicate batch repeats. The time taken to release 50 and $226100 \%$ of the glucose was calculated from the results.

\subsection{In vitro mucoadhesion}

229 Adhesion experiments were carried out using a texture analyser (TA) with a $23010 \mathrm{~mm}$ cylindrical probe (on a TA-XT plus, Stable Micro Systems, UK). Porcine 231 tongues were collected from a local butcher (P D Jennings, Hurst, UK) less 232 than 24 hours after slaughter. They were stored on ice whilst the majority of 233 muscle and connective tissue was removed leaving a thin section of the 234 surface mucosa. These sections were stored at $-20^{\circ} \mathrm{C}$ until required when 235 they were thawed in the fridge for 3 hours before use.

237 Each area of the tongue was cut into $1 \mathrm{~cm}^{2}$ sections and secured on the 238 bottom platform of the TA. The film sample to be tested was stuck to the 
239 probe with double-sided sticky tape. Before each experiment, the tongue

240 tissue section was conditioned with $100 \mu \mathrm{L}$ of $\mathrm{AS}$ and incubated at $37^{\circ} \mathrm{C}$. The

241 contact time between the probe and the tissue was 60 seconds before pulling

242 apart with a removal speed of $1 \mathrm{~mm} / \mathrm{s}$.

243

$244 \quad$ 2.7. In vivo retention

245 The study was given a favourable opinion for conduct by the University of

246 Reading, School of Chemistry, Food and Pharmacy (study number 27/15).

247 Five volunteers (3 males and 2 females, age range 23-30) were asked to

248 place a film sample on their tongue and keep it between the tongue and roof

249 of their mouth for the duration of the experiment. They were instructed to treat 250 the film like a hard candy with some manipulation by the tongue. The

251 experiment was timed and volunteers were asked to note the time (s) when 252 the film began to adhere, when the adherence ceased and when the film 253 dissolved. They were also asked where in the mouth the film adhered to. 254 Adherence was noted as an inability to move the film with their tongue.

\subsection{Sensory perception}

257 Time intensity; profiling involves trained sensory panellists continuously 258 recording the intensity of one or two attributes over a specified time. This 259 enables perception to be captured during consumption and can be 260 summarised as parameters such as onset, persistence and duration. Over a 261 period of three weeks, 8 trained panellists from the University of Reading 262 Sensory Science Centre panel scored each of the film samples in duplicate. 263 There were 12 samples in total. For each polysaccharide, films were made 
264 with either glucose alone, vanillin alone or glucose with vanillin. Each week 265 was used for one set of polysaccharide films. For example, in week 1 the 266 glucose only films were scored, in a balanced order, for sweetness over time.

268 Training took place before each scoring week to familiarise the panel to the 269 samples and the time intensity protocol. Each film was presented to the panel 270 and a discussion of the different flavour release behaviours for each of them 271 took place. During these sessions, the panel were given 3 standards for both 272 glucose and vanillin. Glucose standards were $8 \%, 4 \%$ and $2 \%$, and aroma 273 samples were $0.02 \%, 0.01 \%$, and $0.005 \%$. The panellists decided where 274 these standards scored on the line scale with their strongest standard 275 representing 100 on a standard 100-point scale. These standards were given 276 to the panellists at the start of each scoring sessions to re-familiarise them 277 with the standard intensities.

279 Panellists were trained on single and dual attribute time intensity scoring 280 using Compusense@hand software (Ontario, Canada) and feedback was 281 given to those who were not showing good reproducibility. The time intensity 282 test lasted for 5 minutes, which was the agreed amount of time that the 283 panellists could concentrate for without fatigue or boredom. The attributes 284 scored were sweet for glucose only films, vanilla for aroma only films and both 285 sweet and vanilla for the combined films. Panellists were also trained on how 286 to manipulate the sample in the mouth. They were asked to gently rub the film 287 between the tongue and roof of the mouth to facilitate flavour release. 
288 Panellists were instructed to treat each sample the same way to avoid biasing 289 release.

291 Each week the panellists were given a training session on the first day 292 followed by two days of scoring the samples. Four samples were served 293 monadically, in a petri dish, in a balanced order with individual blinding codes 294 each day with the duplicate being served on a consecutive scoring day. 295 Panellists were provided with isolated sensory booths, computers with 296 Compusense Software and warm water for palate cleansing. There was a 2297 minute delay between samples to allow for palate cleansing. Time intensity 298 curves were produced for each panellist and each sample in duplicate.

\subsection{Statistical analysis}

301 One way or two way repeated measures ANOVA (rmANOVA) was used for 302 the appropriate test. Bonferroni or Tukey's HSD corrections were used on 303 pairwise analysis to account for multiple comparisons, at a significance level 304 of $p \leq 0.05$.

305

3. Results \& Discussion

\subsection{Film characteristics}

308 A range of standard methods were used to characterise the polymeric films 309 (Morales \& McConville, 2011; Nair et al., 2013). Each film was measured for 310 thickness, water activity $\left(\mathrm{aw}_{\mathrm{w}}\right)$, glucose release, and swelling / disintegration 311 times (Table 3). 
313 The thickness of the films varied between the different polysaccharides and

314 between the films with and without glucose. The order of film thickness was $315 \mathrm{HPMC}>\mathrm{HCMC}>\mathrm{LCMC}>\mathrm{PUL}$. This is not surprising as HPMC and HCMC were 316 higher viscosity grades than LCMC and PUL and therefore will occupy more 317 space, retain more water and form thicker films. Glucose films were thicker 318 than those without glucose, which was expected, as the glucose was in 319 addition to the polysaccharides. The thickness of a film will impact the 320 dissolution rate as a thicker film will have a smaller surface area to volume 321 ratio and this can slow water uptake from the surrounding medium. This will 322 impact mucoadhesion as hydration of the dosage form is integral for polymer 323 mucin interactions to occur.

PUL and LCMC films fully dissolved after a similar time; however, LCMC films 326 swelled more before beginning to disintegrate (Table 3 \& Figure $\mathrm{S} 1$ ). This is 327 because LCMC is more viscous than PUL (table 1) and possesses ionic 328 groups, which interact strongly with water molecules due to the higher osmotic pressure induced by the high entropy of the counter-ions. LCMC and HCMC 330 films swelled considerably more than the non-ionic, PUL and HPMC films with 331 relation to their disintegration time. The carboxymethyl cellulose films 332 absorbed more water, forming a swollen gel-like layer, before beginning to 333 degrade. HCMC samples took the longest time to dissolve and swelled the 334 most due to their high viscosity. All films without glucose had higher swelling 335 ratios than their glucose containing counterparts and took longer to dissolve. 336 This is because the small, highly hydrophillic glucose molecules contained 337 within the film matrix will quickly dissolve into the surrounding medium, 
338 leaving pores for the water molecules to enter, effectively increasing the 339 surface area of the film.

341 The glucose release from the films followed a similar pattern to the dissolution 342 rates. PUL and LCMC released glucose fully after 7.0 and $7.8 \mathrm{~min}$ 343 respectively, followed by HPMC (186 min) and then HCMC (300 min). HPMC 344 quickly released $50 \%$ of the total glucose in the film over a mean of 14 345 minutes. This fast initial release is most likely due to crystallisation of the 346 glucose molecules on the outside of the film. This was visually observed, as 347 these films were cloudy with a fine powder covering them. Furthermore, the 348 HPMC samples took a long time to fully dissolve, most likely due to the high 349 viscosity network it forms which will slow permeation of water molecules. The 350 HCMC released the glucose at a constant rate. The HCMC films swelled 351 considerably so the swollen, surface of the film contained loosely associated 352 polymer chains, which would then allow the glucose molecules to diffuse out 353 and dissolve in the surrounding medium. The increased surface area caused 354 by the high swelling degree of the HCMC films may facilitate glucose release, 355 however, the thick gel layer covering the outer surface of the film may also 356 decrease diffusion by physical entrapment. Additionally, the thick gel layer 357 may prevent matrix disintegration and affect subsequent water uptake when 358 unperturbed (Rodriguez, Bruneau, Barra, Alfonso, \& Doelker, 2000). HPMC 359 did not swell substantially but took a long time to dissolve, therefore the 360 glucose molecules would essentially be trapped in the film matrix until is 361 started to erode. 
Table 3. Characteristics of films

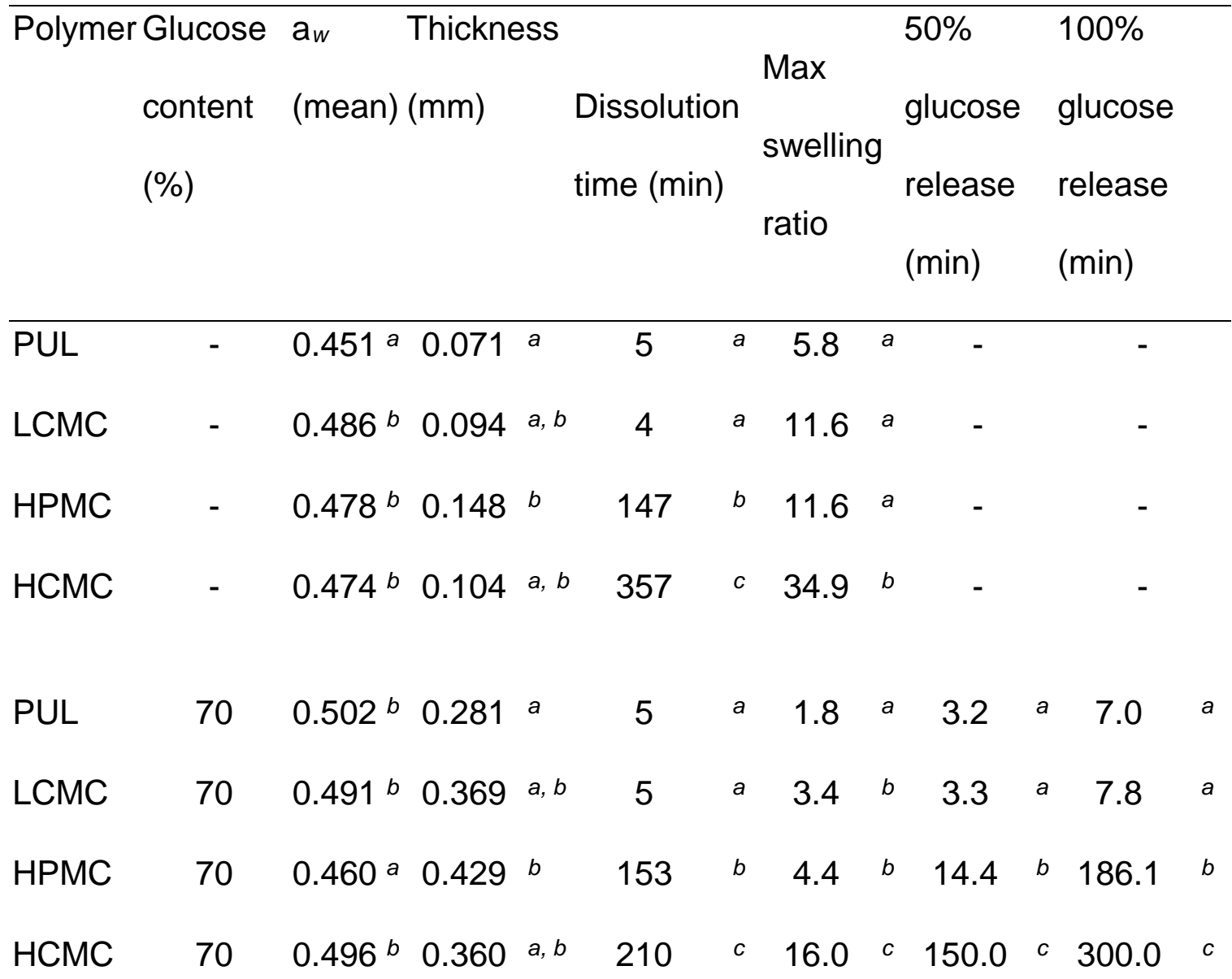

364 Films are separated into those without glucose and those with glucose. Each

365 value is the mean of 6 replications for the measured parameters (2 batch

366 repeats). Mean values within a column and film group not sharing the same

367 letter were significantly different from each other at $p \leq 0.05$ using Tukey's

368 HSD correction.

370 It was expected that changes in flavour perception over time would be

371 influenced by the parameters measured (Table 3). For example, it was

372 hypothesised that PUL films would result in a high intensity flavour that

373 decreased in intensity quickly as they dissolved faster and released glucose

374 quickly. Conversely, it was expected that as the HCMC would slow the 
375 release of glucose and aroma and therefore reduce the initial intensity of 376 flavour but prolong the sensation over time. The results gained from this study 377 are in concordance with the authors expectations.

\subsection{Mucoadhesion in vitro}

380 Two values were obtained from the TA experiments; the maximum force 381 required to separate the probe from the tongue (peak force of detachment) 382 and the area under the curve (total work of adhesion). The mean values for 383 peak force of attachment and total work of adhesion decreased in order of 384 LCMC, HCMC, PUL and HPMC for films without glucose and LCMC, PUL, 385 HCMC and HPMC for films with. In films both with and without glucose the LCMC film was significantly more mucoadhesive than the HPMC film (Figure $1 \mathrm{a} \& \mathrm{~b})$. The films without glucose required a significantly higher force to separate the film from the tissue suggesting a stronger adhesive joint (Figure 1a). This is not surprising as the glucose content was high and therefore the 390 relative amount of polymer in contact with the tissue was smaller. The HPMC 391 films with glucose exerted the lowest total work of adhesion and peak force of 392 detachment (Figure 1). This is probably due to the non-ionic nature of HPMC 393 along with the large molecule size and slow swelling (Table 3 \& Figure S1).

395 Mucoadhesion of solid polymeric substances is dependent on the hydration of 396 the formulation, which will create a polymeric mesh enabling the interactions 397 between polymer and mucin chains. The mucin used in the artificial saliva 398 were PGM purchased from Sigma-Aldrich, which is dehydrated and potentially 399 denatured due to production processes (Kocevar-Nared, Kristl, \& Smid- 
400 Korbar, 1997). Therefore, the interactions that may occur with salivary mucin 401 may not be represented by this commercial mucin. Furthermore, an adhesive 402 joint is formed due to the viscous gel formed between the film and the moist 403 mucosal surface. However, over-hydration of the film will lead to a slippery 404 mucilage being formed and will result in an adhesive joint failure. The swelling 405 ability of a polymeric substance is important for establishing a mucoadhesive 406 bond as this enables polymer chains to be available to interact with the 407 mucosa.

a)

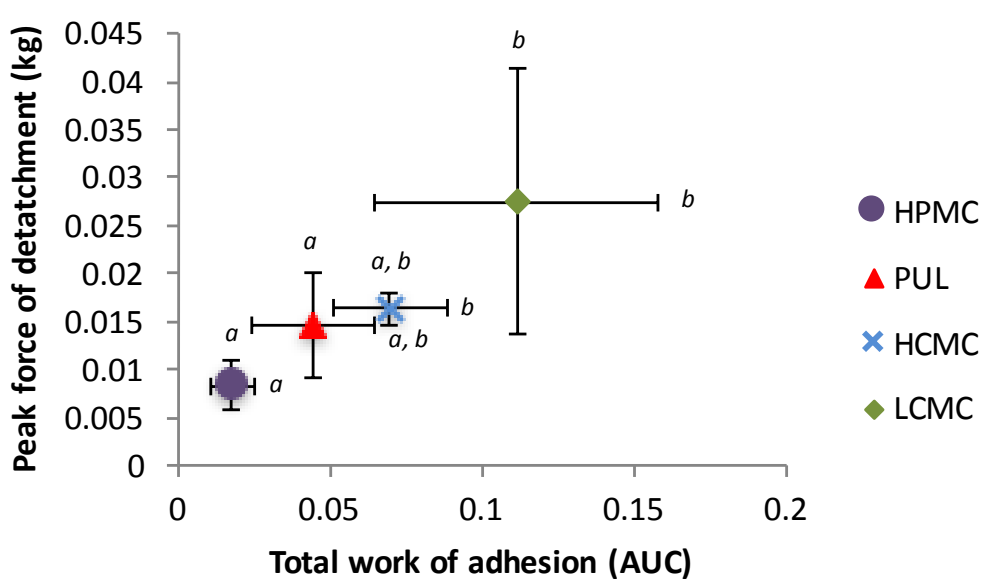

b)

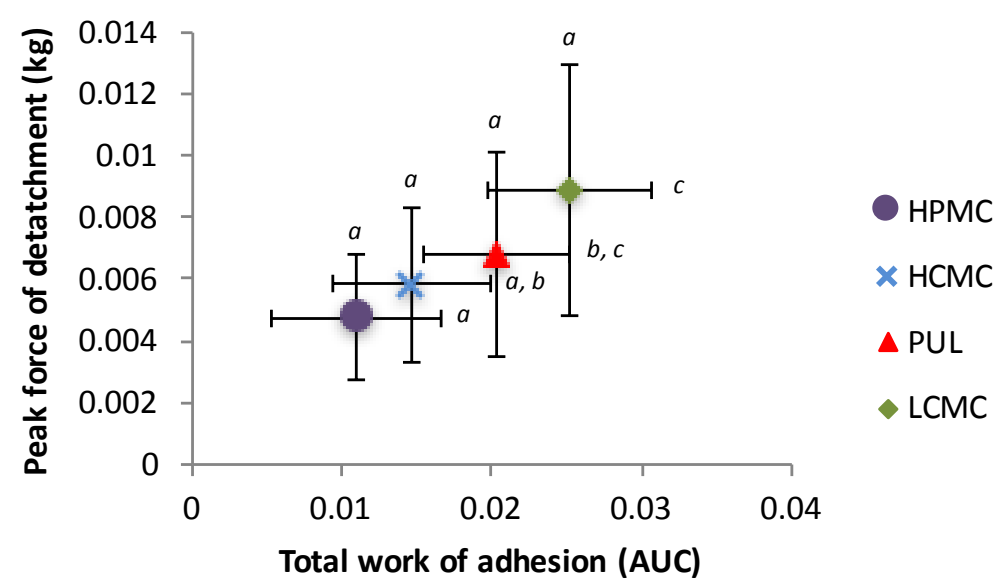

409 Figure 1. Total work of adhesion against the peak force of detachment for

410 films a) without glucose and b) with glucose. Results determined by texture

411 analysis. Data points are means of 6 measurements and error bars are SD.

412 Superscript letters represent statistically different groupings $(p<0.05)$. Letters 
413 on top of the data point refer to the $y$ axis and those to the right hand side

414 refer to the $\mathrm{x}$ axis.

\subsection{Mucoadhesion in vivo}

417 In vivo mucoadhesion experiments were carried out with 5 panellists that were 418 asked to record the following: where the film stuck, for how long and when it 419 dissolved. All films, except for HPMC with glucose, were reported to adhere 420 for the duration of the time that the film was in the mouth (Figure $2 a \& b$ ). 421 Adherence was mainly to the roof of the mouth but also the tongue. The time 422 that the films took to dissolve reflected the in vitro dissolution (Table 3) as 423 PUL and LCMC took the least amount of time to dissolve followed by HPMC 424 then HCMC. For films without glucose, HPMC and HCMC films did not differ 425 in time for dissolution in vivo (Figure 2a) despite the difference in the in vitro 426 test. This is probably due to the participants manipulating the film with their 427 tongue during these experiments, thereby exerting mechanical stress on the 428 film. Therefore, as the HCMC swells and takes up water to produce a gel-like 429 layer, the tongue pressure will remove it and therefore speed up the time of 430 erosion. 

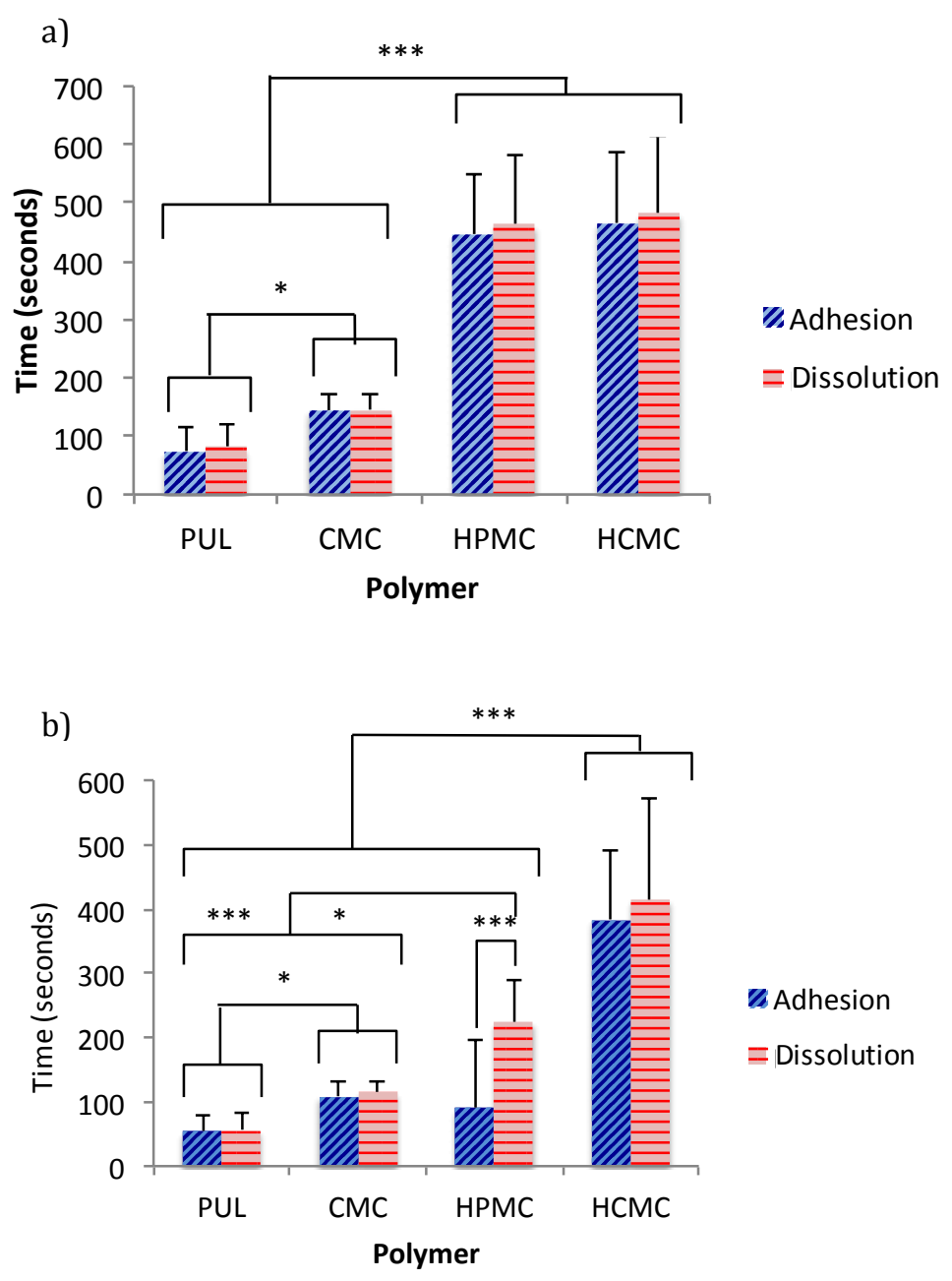

434 Figure 2. In vivo mucoadhesion of a) polymer films without glucose and b) 435 polymer films with glucose. Each bar represents the mean of 10 separate data 436 points, error bars represent standard deviation. $\mathrm{N}=5$ in duplicate. ${ }^{*}=\mathrm{p}<0.05$, $437^{* * *}=p<0.001$.

439 The HPMC films with glucose were reported to adhere for a significantly 440 shorter time than it took to dissolve and 3 out of 5 of the panellists reported 441 that the film did not adhere at all (Figure $2 b$ ). This reflects the in vitro tensile 442 experiments where HPMC was concluded to be significantly less adhesive 443 than the other films. Contrary to these in vitro tensile experiments, HPMC 444 films without glucose were mucoadhesive in the in vivo experiments, with all 
445 panellists reporting adherence after an initial delay. There are two 446 explanations to this. Firstly, AS was used in the in vitro experiments, which 447 contained Sigma-Aldrich PGM as opposed to human salivary mucin. This may 448 affect interactions between the polysaccharide matrix and the saliva due to 449 differences in denaturation states and response to $\mathrm{pH}$. For example, mucin 450 chains must be flexible and uncoiled enough to allow interpenetration with 451 polymer chains. Secondly, the hydration of the oral cavity in vivo may be

452 different to that which was on the porcine tongue in the in vitro experiments.

453 This may have led to a stronger adhesion in vivo, as the film did not become 454 overhydrated.

455

456 The PUL film dissolving and adherence time was significantly quicker for 457 LCMC films in these experiments. The PUL films dissolved on average at 81 458 seconds compared to 145 seconds for the LCMC films during these 459 experiments. This is in contrast to the results obtained from the in vitro 460 dissolution tests (table 3) where they were not significantly different. This 461 difference was expected to have an impact on flavour release from LCMC 462 films compared to PUL. Film thickness is the most likely explanation for the 463 differences observed, LCMC films were thicker than PUL and therefore, when 464 in contact with the moist mucosal surface, will take longer to take up water. To 465 properly assess the impact of polysaccharide type on dissolution times, the 466 thickness of the films would need to be matched.

468 3.4. Perception of tastant and aroma from films over time changes depending 469 on polysaccharide used 
470 Panellists produced time intensity curves for each sample and repeat. They

471 continuously scored either sweetness or vanilla, or both attributes at the same

472 time, over the course of 5 minutes using an unstructured line scale. Various 473 parameters were extrapolated from the curves including the area under the 474 curve (AUC), time to maximum intensity ( $\left.T_{\max }\right)$, maximum intensity ( $\left.I_{\max }\right)$, 475 duration of perception, and incline and decline angles (Figure S2). One-way 476 rmANOVA was used for each parameter

\subsubsection{Glucose only films}

479 Time intensity curves were averaged across all panellists and both replicates 480 (Figure 3). The mean sweetness AUC and Imax values for the films decreased 481 in order of PUL $>$ LCMC $>$ HPMC $>$ HCMC with the reverse order for $\mathrm{T}_{\max }$ 482 (Table 4) where PUL was significantly higher than HCMC and higher for all 483 other films for $I_{\max }$. This suggests a fast onset of intensity for PUL and LCMC, 484 which is supported by their larger incline angles compared to HPMC and 485 HCMC. Furthermore, PUL and LCMC decline angles were also larger than the 486 other two film types suggesting a quicker rate of decline. These results were 487 expected as in vitro results (Table 3) show that PUL and LCMC films were 488 faster dissolving and release glucose quicker than HPMC and HCMC films 489 (table 3). Although the total duration of perception was not significantly 490 different between the films, there was a trend that HPMC and HCMC films 491 prolonged the flavour perception compared to PUL and LCMC (see "duration" 492 in table 4). 
494 Regarding mucoadhesion, the HPMC films containing glucose were found to 495 have poor adhesive abilities (Figures 1 \& 2). In the perception experiments 496 panellists were asked not to swallow these films and, therefore, the perception 497 may have been artificially prolonged due to consciously keeping the film in the 498 mouth. During normal consumption in a real food system the material would 499 be chewed into a bolus and, without mucoadhesive ability, it may well be 500 swallowed with the food bolus thereby negating any further release. On the

501 other hand, HCMC films showed strong adhesion (Figures 1 \& 2) and 502 therefore would be more likely to adhere to the oral cavity for longer, 503 prolonging the release.

504

505

\subsubsection{Vanillin only films}

506 For films containing the polysaccharide and vanillin the mean scores for $I_{\max }$ 507 decreased in order of PUL $>$ LCMC $>H P M C>H C M C$ (Table 4, Figure 3b). 508 Where PUL was significantly higher than HCMC. Tmax and AUC were not 509 dependent on polysaccharide type. The duration of perception was longest in 510 the HPMC samples followed by HCMC. This suggests that although the total 511 intensity of perception was the same for each film, the aroma was delivered at 512 a slightly lower intensity for longer in the HPMC and HCMC samples. This is 513 supported by the decline angles being larger for PUL and LCMC samples 514 suggesting the intensity decreased more quickly in these films.

516 To date, the only studies investigating aroma release and perception in food 517 thickened with polysaccharides are in liquid and semi-solid foods. These 518 studies have found confounding results with regard to interactions between 
519 aroma molecules and the food matrix. Arancibia et al. (2011) found that

520 thickener type affected total aroma release from dairy desserts with $\mathrm{CMC}$

521 thickened samples reducing the cumulative release of hydrophobic aroma

522 (linalool) compared to starch. Furthermore, a follow up study by Arancibia,

523 Castro, Jublot, Costell, \& Bayarri (2015) found that thickener type affected

524 both hydrophilic aroma (cis-3-hexen-1-ol) and hydrophobic (linalool) aroma.

525 The CMC thickened dairy desserts reduced the release of both aromas,

526 though it had more of an impact on the hydrophilic compound. Cook, Linforth,

527 et al., (2003) on the other hand found that in-nose measurements of

528 hydrophobic aroma release were not dependent on thickener type or on an

529 increase in viscosity. These studies exemplify the complex behaviour of

530 aroma release and its dependence on the food matrix.

531

532 In this current study, vanillin, a slightly hydrophobic molecule with a $\log P$ of

533 1.2, was used as the aroma. Perception results show that films made with

534 slow dissolving polysaccharides (HPMC and HCMC) reduced the Imax but 535 prolonged the duration of perception. Perception results for the aroma only 536 films were not as distinguishable as the films containing glucose. This may be 537 because the panel found scoring the aroma only films particularly difficult as 538 they contained no tastant along with the aroma, which does not normally 539 occur in food products.

541 3.4.3. Glucose \& vanillin films

542 Dual attribute time intensity was used to simultaneously and continuously 543 monitor sweetness and vanilla attributes over 5 minutes. Results for the 
544 sweetness attribute were similar for the dual attribute and single attribute tests

545 (Table 4, Figure $3 \mathrm{c}$ and $\mathrm{d}$ ). The AUC and $I_{\max }$ were highest for PUL and

546 lowest for HCMC. HPMC and HCMC took longer to reach Tmax compared to

547 PUL and LCMC.

548

549 The AUC for the vanilla attribute did not significantly differ with the different 550 polysaccharides (Table 4). HPMC and HCMC had reduced Imax and increased

551 T max results compared to PUL and LCMC. The total duration of perception 552 was striking in these films with the HCMC averaging 53 seconds longer than

553 PUL. HPMC also increased the duration significantly compared to PUL and

554 LCMC. Although not statistically significant, LCMC followed the trend of 555 prolonging the perception compared to PUL. The incline angles for HPMC and 556 HCMC were, again, smaller than PUL and CMC suggesting a slower rate of 557 onset.

558

559 These results suggest that PUL films give a quick burst of flavour that 560 declines quickly. LCMC films are almost as quick to release as PUL but take 561 somewhat longer to reach $I_{\max }$. HPMC has a slower onset to reach $I_{\max }$ and 562 the perception continues for longer than LCMC and PUL. Finally, HCMC films 563 have the slowest onset with a steady release over time. This is particularly 564 evident for the vanilla attribute, which prolongs the perception for longer than 565 the faster dissolving films.

566

567 Although from this perception data HPMC films appear to give a sustained, 568 medium level intensity of flavour, this formulation was not particularly 
569 mucoadhesive and, therefore, it would most likely be swallowed along with the

570 bolus in a real food system. Participants were instructed not to chew or

571 swallow the film and many suggested that this would have been possible if

572 they were eating normally. However, the other formulations were firmly

573 adhered to the roof or tongue tissue and would not be easily swallowed.

574

575 Table 4. Parameters from time intensity results.

\begin{tabular}{|c|c|c|c|c|c|c|c|c|c|c|c|c|c|}
\hline \multirow[t]{2}{*}{$\begin{array}{l}\text { Film } \\
\text { type }\end{array}$} & \multicolumn{2}{|c|}{ Attribute Polymer } & \multicolumn{2}{|c|}{ AUC } & $\operatorname{Imax}$ & \multicolumn{2}{|c|}{$\begin{array}{c}T_{\max } \\
(\operatorname{secs})\end{array}$} & \multicolumn{2}{|c|}{$\begin{array}{c}\text { Duration } \\
\text { (secs) }\end{array}$} & \multicolumn{2}{|c|}{$\begin{array}{c}\text { Incline } \\
\text { angle } \\
\left(^{\circ}\right)\end{array}$} & \multicolumn{2}{|c|}{$\begin{array}{c}\text { Decline } \\
\text { angle } \\
\left({ }^{\circ}\right)\end{array}$} \\
\hline & \multirow{4}{*}{ Sweet } & PUL & 8410 & $b$ & $91^{d}$ & 22 & $a$ & 200 & $a$ & 73 & $b$ & 30 & $b$ \\
\hline \multirow{3}{*}{ Glucose } & & LCMC & 7468 & $a, b$ & $75^{c}$ & 48 & $b$ & 201 & $a$ & 58 & $b$ & 27 & $b$ \\
\hline & & HPMC & 7126 & $b$ & $54^{b}$ & 61 & $b$ & 231 & $a$ & 38 & $a$ & 20 & $a$ \\
\hline & & HCMC & 4834 & $a$ & $31^{a}$ & 88 & $b$ & 249 & $a$ & 34 & $a$ & 11 & $a$ \\
\hline \multirow{4}{*}{ Aroma } & \multirow{4}{*}{ Vanilla } & PUL & 7291 & $a$ & $68^{b}$ & 41 & $a$ & 196 & $a$ & 57 & $a$ & 25 & $b, c$ \\
\hline & & LCMC & 7154 & $a$ & $59 a, b$ & 40 & $a$ & 195 & $a$ & 50 & $a$ & 28 & $c$ \\
\hline & & HPMC & 7622 & $a$ & $53 a, b$ & 50 & $a$ & 264 & $b$ & 47 & $a$ & 14 & $a$ \\
\hline & & HCMC & 6176 & $a$ & $51^{a}$ & 38 & $a$ & 230 & $a, b$ & 54 & $a$ & 19 & $a, b$ \\
\hline \multirow{8}{*}{$\begin{array}{l}\text { Aroma } \\
\text { and } \\
\text { Glucose }\end{array}$} & \multirow{4}{*}{ Sweet } & PUL & 9154 & $b, c$ & $92^{d}$ & 25 & $a$ & 221 & $a$ & 73 & $c$ & 28 & $b, c$ \\
\hline & & LCMC & 9295 & $c$ & $82^{c}$ & 32 & $a$ & 224 & $a$ & 64 & $b$ & 27 & $c$ \\
\hline & & HPMC & 6661 & $a, b$ & $50^{b}$ & 64 & $b$ & 245 & $a$ & 41 & $a$ & 17 & $a, b$ \\
\hline & & $\mathrm{HCMC}$ & 5864 & $a$ & $36^{b}$ & 64 & $b$ & 266 & $a$ & 34 & $a$ & 12 & $a$ \\
\hline & \multirow{4}{*}{ Vanilla } & PUL & 9499 & $a$ & $87^{b}$ & 29 & $a$ & 239 & $a$ & 67 & $b$ & 21 & $a$ \\
\hline & & LCMC & 10957 & & $82^{b}$ & 35 & $a$ & 254 & $a$, & 67 & $b$ & 23 & $a$ \\
\hline & & HPMC & 10081 & & $56^{a}$ & 54 & $\begin{array}{l}a, \\
b\end{array}$ & 276 & $b$ & 46 & $a$ & 14 & $a$ \\
\hline & & HCMC & 10770 & & $54^{a}$ & 73 & $b$ & 292 & $b$ & 43 & $a$ & 16 & $a$ \\
\hline
\end{tabular}


5768 panellists scored each sample in duplicate therefore each result is the mean 577 of 16 separate results. Statistical analysis was done for each attribute 578 separately comparing the different polysaccharides. Different letters represent 579 significantly different groupings for each set of data.
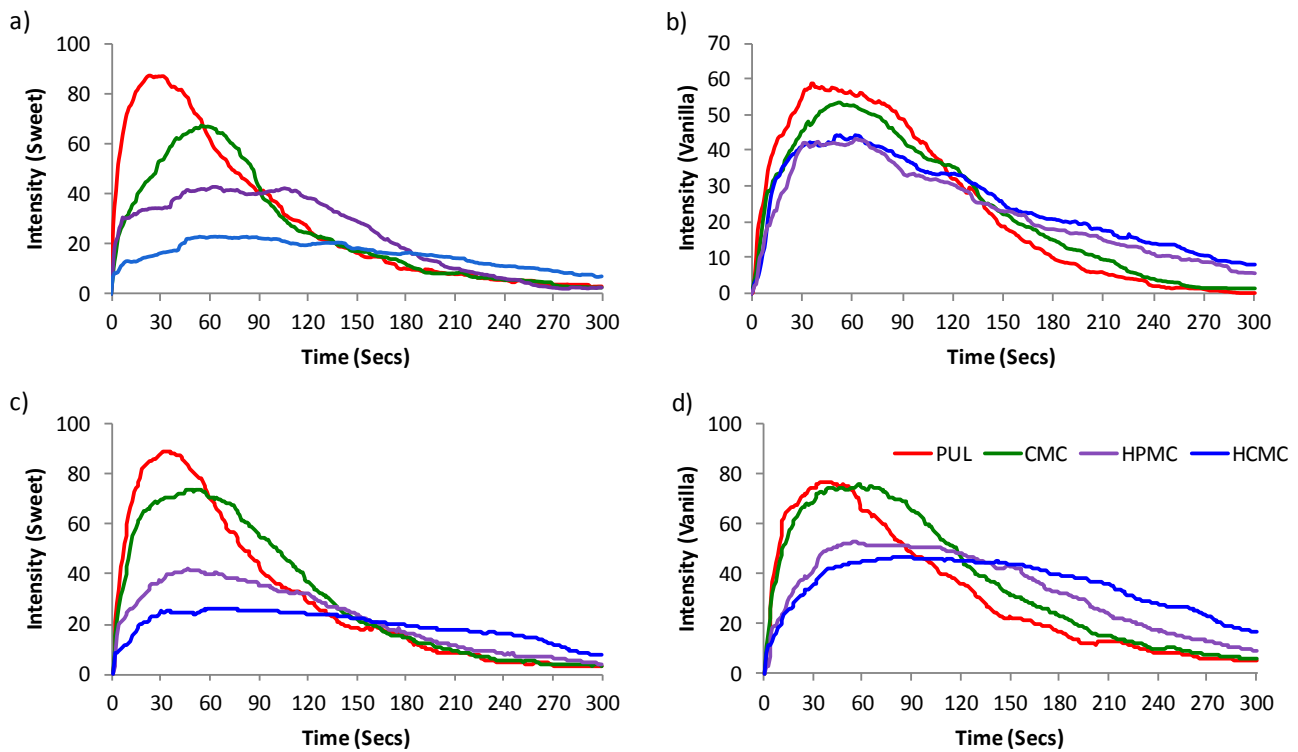

580

581 Figure 3. A panel of 8 trained panellists scored different polysaccharide films

582 in duplicate for either sweetness of vanilla perception over time. Time intensity

583 curves for a) glucose only films, b) vanillin only films were produced from

584 single attribute time intensity tests. Dual attribute time intensity tests produced

585 the curves for glucose and vanillin films in c) and d).

586

\subsection{Comparing perception results to in vivo dissolution}

588 During the in vivo experiments where participants were asked to record the

589 adhesion and dissolution of the films, PUL was reported to dissolve after an

590 average of 57 seconds. When comparing these timings to the perception data

591 it is clear that perception of flavour is continuing after the film has completely

592 dissolved (Table 4 \& Figure 3). There are two explanations for this. Firstly, the

593 glucose and aroma molecules may still be present at the respective receptors, 
594 thereby initiating a response. Secondly, as the intensity of sweetness was 595 very high, an adaptation type response could occur where the sweet signal is

596 switched on for a longer time even after the stimulus has gone.

598 The physiological differences between participants were not collected for the 599 in vivo mucoadhesion nor the sensory perception experiments. Factors such 600 as salivary flow and constituents varies between individuals (Fenoli601 Palomares et al., 2004) and will therefore impact the mucoadhesive strength 602 and rate of film dissolution. Despite not adding these covariates in analysis, 603 there were still significant results gained from the experiments.

604

605

\subsection{Comparisons between different film types}

606 Time intensity results were compared between 5 panellists who were 607 consistent for both experiments. The AUC for the vanilla attribute differed 608 between films with and without glucose (Figure 4). Significant increases in the 609 total perception intensity (AUC) of vanilla were observed for LCMC, HPMC 610 and HCMC films containing vanillin plus glucose compared to those without 611 glucose.

613 During single attribute time intensity, the attribute is scored horizontally but 614 during dual attribute, one must be scored vertically. The vanilla attribute was 615 scored vertically in the dual attribute tests, which may have affected the 616 results. Duizer, Bloom, \& Findlay, (1995) investigated this issue and found 617 that scoring an attribute vertically lead to approximately $13 \%$ increase in 
618 scores. However, as the increase is more substantial it is unlikely this is the 619 only factor.

621 A more likely explanation is that the presence of glucose in the films 622 enhanced the aroma through cross modality (D. J. Cook et al., 2003; Niimi, 623 Eddy, Overington, Heenan, et al., 2014; Niimi, Eddy, Overington, Silcock, et 624 al., 2014). $T_{\max }$ was also significantly $(p<0.05)$ increased for vanillin in the 625 HCMC films going from 26 to 89 seconds (Figure S3). This suggests that 626 when glucose was present the perception of aroma had a slower onset, which 627 lasted for longer and was sustained.

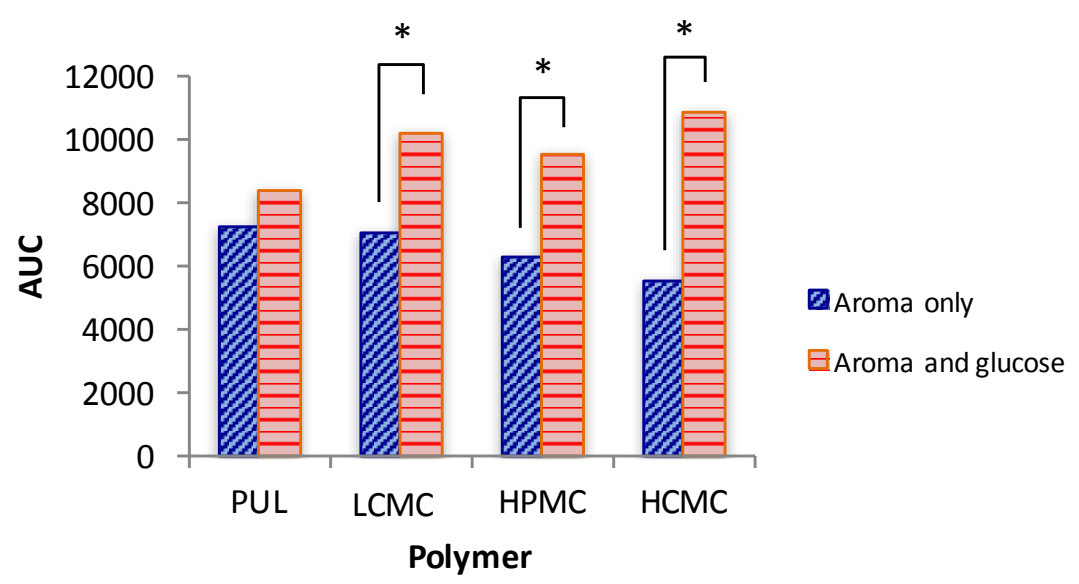

629 Figure 4. Comparisons of the area under the curve for the vanilla attribute of 630 films with and without glucose. * denotes significant differences $p=<0.05$ 631 using Bonferroni correction.

632

4. Conclusions

634 This study has shown that polysaccharides affect the retention, release and 635 perception of flavour compounds, dependant on the physicochemical 636 properties of the polysaccharide matrix. The viscosity and swelling ability of 
637 the polysaccharide influences the release of flavour molecules from the 638 matrix. This in turn has an impact on the flavour perception. Fast dissolving 639 polysaccharides resulted in a quick burst of flavour at high intensity that 640 tapered more quickly whereas slow dissolving films gave a slower onset and a 641 more consistent release over time. The mucoadhesive ability of the films will 642 influence how long the matrix stays in the mouth whilst releasing the flavour 643 compounds before being swallowed. Furthermore, in line with previous 644 literature, this study shows that aroma intensity is dependent on the 645 perception of a congruent tastant, giving more evidence for cross modal 646 interactions.

648 The mucoadhesive nature of some of the polysaccharides tested will have an 649 effect on flavour delivery over time as those that adhere to the oral cavity will 650 continue to release flavour whilst those that are not mucoadhesive will be 651 swallowed. This study investigated flavour release from very simple food 652 matrices, polysaccharide films; of course in a real food there will be many 653 other food components that could affect flavour release. However, this study 654 provides some fundamental understanding of how different polysaccharide 655 matrices affect flavour release. Results from this study can be used to inform 656 the food industry of the impact that the addition of these polysaccharides can 657 have on temporal flavour perception. Possible applications include topical 658 coatings, confectionary, low fat and low sugar foods. However, there is a need 659 for further research into this area to understand the full impact on the 660 organoleptic properties of foods. 
662 Acknowledgements

663 This work has been funded as part of BBSRC CASE studentship 664 (BB/K012029/1). McCormick (UK) Ltd is also thanked for contribution of 665 funding to BB/K012029/1 studentship.

666

667

668

669

670

671

672

673

674

675

676

677

678

679

680

681

682

683

684

685

686 
References

688

689

690

691

692

693

694

695

696

697

698

699

700

701

702

703

704

705

706

707

708

709

710

711

Andrews, G. P., Laverty, T. P., \& Jones, D. S. (2009). Mucoadhesive polymeric platforms for controlled drug delivery. European Journal of Pharmaceutics and Biopharmaceutics : Official Journal of Arbeitsgemeinschaft Für Pharmazeutische Verfahrenstechnik e.V, 71(3), 505-18. http://doi.org/10.1016/j.ejpb.2008.09.028

Arancibia, C., Castro, C., Jublot, L., Costell, E., \& Bayarri, S. (2015). Colour, rheology, flavour release and sensory perception of dairy desserts. Influence of thickener and fat content. LWT - Food Science and Technology, 62(1), 408-416. http://doi.org/10.1016/j.Iwt.2014.08.024

Arancibia, C., Jublot, L., Costell, E., \& Bayarri, S. (2011). Flavor release and sensory characteristics of o/w emulsions. Influence of composition, microstructure and rheological behavior. Food Research International, 44(6), 1632-1641. http://doi.org/10.1016/j.foodres.2011.04.049

Bayarri, S., Taylor, A. J., \& Hort, J. (2006). The Role of Fat in Flavor Perception: Effect of Partition and Viscosity in Model Emulsions. Journal of Agricultural and Food Chemistry, 54, 8862-8868.

Bull, S. P., Hong, Y., Khutoryanskiy, V. V., Parker, J. K., Faka, M., \& Methven, L. (2015). Whey protein mouth drying influenced by thermal denaturation. Food Quality and Preference, 56, 233-240. http://doi.org/10.1016/j.foodqual.2016.03.008

Cook, D. J., Linforth, R. S. T., \& Taylor, A. J. (2003). Effects of hydrocolloid thickeners on the perception of savory flavors. Journal of Agricultural and Food Chemistry, 51(10), 3067-3072. http://doi.org/10.1021/jf0211581

Cook, S. L., Bull, S. P., Methven, L., Parker, J. K., \& Khutoryanskiy, V. V. 

(2017). Mucoadhesion: A food perspective. Food Hydrocolloids, 72, 281296.

714 Cook, S. L., Woods, S., Methven, L., Parker, J. K., \& Khutoryanskiy, V. V.

715 (2018). Mucoadhesive polysaccharides modulate sodium retention,

716 release and taste perception. Food Chemistry, 240, 482-489. http://doi.org/10.1016/j.foodchem.2017.07.134

718

719

720

De Hoog, E. H. A., Prinz, J. F., Huntjens, L., Dresselhuis, D. M., \& Van Aken, G. A. (2006). Lubrication of oral surfaces by food emulsions: The importance of surface characteristics. Journal of Food Science, 71(7). http://doi.org/10.1111/j.1750-3841.2006.00140.x

Dresselhuis, D. M., van Aken, G. A., de Hoog, E. H. A., \& Martien, A. C. S. (2008). Direct observation of adhesion and spreading of emulsion droplets at solid surfaces. Soft Matter, 4(4), 1079-1085. http://doi.org/10.1039/b800106e

Duizer, L. M., Bloom, K., \& Findlay, C. J. (1995). The effect of line orientation on the recording of time-intensity perception of sweetener solutions. Food Quality and Preference, 6(2), 121-126. http://doi.org/10.1016/09503293(94)00021-M

Esteban-Fernández, A., Rocha-Alcubilla, N., Muñoz-González, C., MorenoArribas, M. V., \& Pozo-Bayón, M. Á. (2016). Intra-oral adsorption and release of aroma compounds following in-mouth wine exposure. Food Chemistry, 205, 280-288. http://doi.org/10.1016/j.foodchem.2016.03.030

Fenoli-Palomares, C., Munoz-Montagud, J. V, Sanchiz, V., Herreros, B., Hernandez, V., Minguez, M., \& Benages, A. (2004). Unstimulated salivary flow rate, $\mathrm{pH}$ and buffer capacity of saliva in healthy volunteers. Revista 
Española de Enfermedades Digestivas, 96(11), 773-783. Retrieved from http://scielo.isciii.es/pdf/diges/v96n11/original4.pdf

739 Gherman, S., Zavastin, D., Ochiuz, L., Biliuta, G., \& Coseri, S. (2016). Enalapril maleate loaded pullulan film for mucoadhesive buccal drug

Gibbins, H. L., \& Carpenter, G. H. (2013). Alternative mechanisms of astringency - What is the role of saliva? Journal of Texture Studies, 44(5),

Hollowood, T. A., Linforth, R. S. T., \& Taylor, A. J. (2002). The effect of Hilal Y, Ç., Gudjónsdóttir, M., Meier, S., Duus, J. Ø., Lee, S., \& Chronakis, I. Heilig, A., Heimpel, K., Sonne, A., Schieberle, P., \& Hinrichs, J. (2016). An approach to adapt aroma in fat-free yoghurt systems: Modelling and transfer to pilot scale. International Dairy Journal, 56, 101-107. http://doi.org/10.1016/j.idairyj.2016.01.011

S. (2015). Spectroscopic studies of the interactions between betalactoglobulin and bovine submaxillary mucin. Food Hydrocolloids. http://doi.org/10.1016/j.foodhyd.2015.04.026

761 Izutsu, T., Taneya, S., Kikuchi, E., \& Sone, T. (1981). Effect of viscosity on 
perceived sweetness intensity of sweetened sodium carboxymethylcellulose solutions. Journal of Texture Studies, 12(2), 259273.

765

766

Jabbari, E., Wisniewski, N., \& Peppas, N. A. (1993). Evidence of mucoadhesion by chain interpenetration at a poly (acrylic acid)/mucin interface using ATR-FTIR spectroscopy. Journal of Controlled Release. http://doi.org/10.1016/0168-3659(93)90109-I

Juliano, C., Gavini, E., Cossu, M., Bonferoni, M. C., \& Giunchedi, P. (2004). Mucoadhesive alginate matrices containing sodium carboxymethyl starch for buccal delivery: in vitro and in vivo studies. Journal of Drug Delivery Science and Technology, 14(2), 159-163. http://doi.org/10.1016/S17732247(04)50029-1

Kaur, A., \& Kaur, G. (2012). Mucoadhesive buccal patches based on interpolymer complexes of chitosan-pectin for delivery of carvedilol. Saudi Pharmaceutical Journal, 20(1), 21-27. http://doi.org/10.1016/j.jsps.2011.04.005

Keršiene, M., Adams, A., Dubra, A., Kimpe, N. De, \& Leskauskaite, D. (2008). Interactions between flavour release and rheological properties in model custard desserts: Effect of starch concentration and milk fat. Food Chemistry, 108(4), 1183-1191. http://doi.org/10.1016/j.foodchem.2007.11.011

Kocevar-Nared, J., Kristl, J., \& Smid-Korbar, J. (1997). Comparative rheological investigation of crude gastric mucin and natural gastric mucus. Biomaterials, 18(9), 677-81. Retrieved from http://www.ncbi.nlm.nih.gov/pubmed/9151999 
Kokini, J. L., Bistany, K., Poole, M., \& Stier, E. (1982). Use of mass transfer

788

789

790

791

792

793

794

795

796

797

798

799

800

801

802

803

804

805

806

807

808

809

810

811 theory to predict viscosity-sweetness interactions of fructose and sucrose solutions containing tomato solids. J. Texture Studies, 13, 187-200.

Kora, E. P., Souchon, I., Latrille, E., Martin, N., \& Marin, M. (2004).

Composition rather than viscosity modifies the aroma compound retention of flavored stirred yogurt. Journal of Agricultural and Food Chemistry, 52(10), 3048-56. http://doi.org/10.1021/jf034597o

Kuo, W.-Y., \& Lee, Y. (2014). Effect of Food Matrix on Saltiness PerceptionImplications for Sodium Reduction. Comprehensive Reviews in Food Science and Food Safety, 13(5), 906-923. http://doi.org/10.1111/15414337.12094

Madsen, K. D., Sander, C., Baldursdottir, S., Pedersen, A. M. L., \& Jacobsen, J. (2013). Development of an ex vivo retention model simulating bioadhesion in the oral cavity using human saliva and physiologically relevant irrigation media. International Journal of Pharmaceutics, 448(2), 373-81. http://doi.org/10.1016/j.ijpharm.2013.03.031

Malone, M. E., Appelqvist, I. A. M., \& Norton, I. T. (2003). Oral behaviour of food hydrocolloids and emulsions. Part 1. Lubrication and deposition considerations. Food Hydrocolloids, 17(6), 763-773. http://doi.org/10.1016/S0268-005X(03)00097-3

Morales, J. O., \& McConville, J. T. (2011). Manufacture and characterization of mucoadhesive buccal films. European Journal of Pharmaceutics and Biopharmaceutics : Official Journal of Arbeitsgemeinschaft Für Pharmazeutische Verfahrenstechnik e.V, 77(2), 187-99. http://doi.org/10.1016/j.ejpb.2010.11.023 
Nair, A. B., Kumria, R., Harsha, S., Attimarad, M., Al-Dhubiab, B. E., \& Alhaider, I. A. (2013). In vitro techniques to evaluate buccal films. Journal of Controlled Release, 166(1), 10-21. http://doi.org/10.1016/j.jconrel.2012.11.019

Niimi, J., Eddy, A. I., Overington, A. R., Heenan, S. P., Silcock, P., Bremer, P. J., \& Delahunty, C. M. (2014). Aroma-taste interactions between a model cheese aroma and five basic tastes in solution. Food Quality and Preference, 31(1), 1-9. http://doi.org/10.1016/j.foodqual.2013.05.017

Niimi, J., Eddy, A. I., Overington, A. R., Silcock, P., Bremer, P. J., \& Delahunty, C. M. (2014). Cross-modal interaction between cheese taste and aroma. International Dairy Journal, 39(2), 222-228. http://doi.org/10.1016/j.idairyj.2014.07.002

Peppas, N., \& Huang, Y. (2004). Nanoscale technology of mucoadhesive interactions. Advanced Drug Delivery Reviews, 56(11), 1675-1687. http://doi.org/10.1016/j.addr.2004.03.001

Richardson, J. C., Dettmar, P. W., Hampson, F. C., \& Melia, C. D. (2004). Oesophageal bioadhesion of sodium alginate suspensions: particle swelling and mucosal retention. European Journal of Pharmaceutical Sciences : Official Journal of the European Federation for Pharmaceutical Sciences, 23(1), 49-56. http://doi.org/10.1016/j.ejps.2004.05.001

Rodríguez-Bencomo, J. J., Muñoz-González, C., Andújar-Ortiz, I., MartínÁlvarez, P. J., Moreno-Arribas, M. V., \& Pozo-Bayón, M. Á. (2011). Assessment of the effect of the non-volatile wine matrix on the volatility of typical wine aroma compounds by headspace solid phase microextraction/gas chromatography analysis. Journal of the Science of 
838 Rodriguez, C. F., Bruneau, N., Barra, J., Alfonso, D., \& Doelker, E. (2000). Hydrophilic cellulose derivatives as drug delivery carriers: influence of the substitution type on the properties of compressed matrix tablets. Retrieved from http://books.google.com/books?id=paEzHRHIXE4C\&pgis=1

Sánchez-López, J. A., Ziere, A., Martins, S. I. F. S., Zimmermann, R., \& Yeretzian, C. (2016). Persistence of aroma volatiles in the oral and nasal cavities: real-time monitoring of decay rate in air exhaled through the

Satishbabu, B. K., \& Srinivasan, B. P. (2008). Preparation and evaluation of buccoadhesive films of atenolol. Indian Journal of Pharmaceutical Sciences. http://doi.org/10.4103/0250-474X.41451

Scherf, K. A., Pflaum, T., Koehler, P., \& Hofmann, T. (2015). Salt taste perception in hydrocolloid systems is affected by sodium ion release and mechanosensory-gustatory cross-modal interactions. Food Hydrocolloids, 51, 486-494. http://doi.org/10.1016/j.foodhyd.2015.05.043

Secouard, S., Malhiac, C., Grisel, M., \& Decroix, B. (2003). Release of limonene from polysaccharide matrices: Viscosity and synergy effect. Food Chemistry, 82(2), 227-234. http://doi.org/10.1016/S03088146(02)00518-6

Semalty, M., Semalty, A., Kumar, G., \& Juyal, V. (2008). Development of Mucoadhesive Buccal Films of Glipizide. International Journal of Pharmaceutical Sciences and Nanotechnology, 1(2), 184-190. 
862 Shaikh, R., Singh, T. R. R., Garland, M. J., Woolfson, A. D., \& Donnelly, R. F. (2011). Mucoadhesive drug delivery systems. Journal of Pharmacy \& Bioallied Sciences, 3(1), 89-100. http://doi.org/10.4103/0975-7406.76478

Smart, J. D. (2005). The basics and underlying mechanisms of mucoadhesion. Advanced Drug Delivery Reviews, 57(11), 1556-68. http://doi.org/10.1016/j.addr.2005.07.001

Smart, J. D. (2014). Theories of Mucoadhesion - Mucoadhesive Materials and Drug Delivery Systems. In V. V. Khutoryanskiy (Ed.), . London: Jon Wiley \& Sons Ltd.

Stevenson, R. J., \& Mahmut, M. K. (2011). Experience dependent changes in odour-viscosity perception. Acta Psychologica, 136(1), 60-66. http://doi.org/10.1016/j.actpsy.2010.10.001

Thirawong, N., Nunthanid, J., Puttipipatkhachorn, S., \& Sriamornsak, P. (2007). Mucoadhesive properties of various pectins on gastrointestinal mucosa: an in vitro evaluation using texture analyzer. European Journal of Pharmaceutics and Biopharmaceutics : Official Journal of Arbeitsgemeinschaft Für Pharmazeutische Verfahrenstechnik e.V, 67(1),

883 Yehia, S. A., El-Gazayerly, O. N., \& Basalious, E. B. (2008). Design and in 884 vitro/in vivo evaluation of novel mucoadhesive buccal discs of an 885 886 132-40. http://doi.org/10.1016/j.ejpb.2007.01.010

Withers, C. A., Cook, M. T., Methven, L., Godney, M. A., \& Khutoryanskiy, V. V. (2013). Investigation of milk proteins binding to the oral mucosa. Food \& Function. http://doi.org/10.1039/C3FO60291E antifungal drug: relationship between swelling, erosion, and drug release. AAPS PharmSciTech, 9(4), 1207-1217. http://doi.org/10.1208/s12249- 
Yehia, S. A., El-Gazayerly, O. N., \& Basalious, E. B. (2009). Fluconazole

889 mucoadhesive buccal films: in vitro/in vivo performance. Current Drug

890 Delivery, 6(1), 17-27. http://doi.org/10.2174/156720109787048195

891 